\title{
Genome-Wide Association Analysis of the Genetic Basis for Sheath Blight Resistance in Rice
}

\author{
Fan Zhang, Dan Zeng, Cong-Shun Zhang, Jia-Ling Lu, Teng-Jun Chen, Jun-Ping Xie and Yong-Li Zhou* (1)
}

\begin{abstract}
Background: Sheath blight (ShB), caused by Rhizoctonia solani Kühn, is one of the most destructive rice diseases. Developing ShB-resistant rice cultivars represents the most economical and environmentally sound strategy for managing ShB.

Results: To characterize the genetic basis for ShB resistance in rice, we conducted association studies for traits related to ShB resistance, namely culm length $(C L)$, lesion height $(L H)$, and relative lesion height $(R L H)$. Combined a single locus genome-wide scan and a multi-locus method using 2,977,750 single-nucleotide polymorphisms to analyse 563 rice accessions, we detected 134, 562, and 75 suggestive associations with CL, LH, and RLH, respectively. The adjacent signals associated with RLH were merged into 27 suggestively associated loci (SALs) based on the estimated linkage disequilibrium blocks. More than 44\% of detected RLH-SALs harboured multiple QTLs/genes associated with ShB resistance, while the other RLH-SALs were putative novel ShB resistance loci. A total of 261 ShB resistance putative functional genes were screened from 23 RLH-SALs according to bioinformatics and haplotype analyses. Some of the annotated genes were previously reported to encode defence-related and pathogenesis-related proteins, suggesting that quantitative resistance to ShB in rice is mediated by SA- and JAdependent signalling pathways.
\end{abstract}

Conclusions: Our findings may improve the application of germplasm resources as well as knowledge-based ShB management and the breeding of ShB-resistant rice cultivars.

Keywords: Sheath blight resistance, Germplasm, GWAS, Rice

\section{Background}

Sheath blight (ShB), caused by Rhizoctonia solani Kühn ( $R$. solani), is one of the most destructive rice diseases (Zheng et al. 2013). This disease, which is prevalent in East Asia and the southern USA (Chen et al. 2012; Prasad and Eizenga 2008), results in heavy rice yield losses when nitrogen fertilizers are extensively applied (Savary et al. 1995). $R$. solani is a soil-borne hemibiotrophic pathogen (Kouzai et al. 2018) that survives as sclerotia or mycelia in the debris of host plants during its necrotrophic phase. To date, no major ShB resistance genes or rice cultivars exhibiting complete resistance to $R$. solani have been reported, likely because of the

\footnotetext{
*Correspondence: zhouyongli@caas.cn

Institute of Crop Sciences/The National Key Facility for Crop Gene Resources and Genetic Improvement, Chinese Academy of Agricultural Sciences, 12 South Zhong-Guan-Cun Street, Haidian District, Beijing 100081, China
}

polygenic nature of $\mathrm{ShB}$ resistance. The application of chemicals remains the major method for controlling rice ShB. However, the overuse of chemical fungicides contributes to increased health risks and environmental problems (Zeng et al. 2011). Therefore, developing ShBresistant rice cultivars represents the most economical and environmentally sound strategy for managing $\mathrm{ShB}$ (Liu et al. 2009).

Following the detection of the first quantitative trait locus (QTL) for ShB resistance in rice (Li et al. 1995), more than 60 QTLs conferring ShB resistance have been detected among the 12 rice chromosomes based on biparental genetic mapping populations (Taguchi-Shiobara et al. 2013; Wen et al. 2015; Yadav et al. 2015; Zeng et al. 2011; Zeng et al. 2015). Of these, only qSBR11-1 and $q S B-11^{\mathrm{LE}}$ have been fine-mapped (Channamallikarjuna et al. 2010; Zuo et al. 2013). Additionally, a rice 
chitinase gene (LOC_Os11g47510), which was cloned from the qSBR11-1 region of an $R$. solani-tolerant rice line (Tetep), was functionally validated by the genetic transformation of an ShB-susceptible japonica rice line (Taipei 309) (Richa et al. 2017). Moreover, some major QTLs associated with ShB resistance, such as $q S B-9^{\mathrm{Tq}}$, have been used in breeding programmes (TaguchiShiobara et al. 2013; Zuo et al. 2008). Pyramiding diverse ShB resistance alleles from QTLs differing in their level of moderate resistance by marker-assisted selection can efficiently enhance the resistance of rice to R. solani (Hossain et al. 2016; Yadav et al. 2015). However, the resistance level of a near-isogenic line containing three ShB resistance alleles was not significantly higher than that of a line containing two ShB resistance alleles (Zeng et al. 2011).

A genome-wide association study (GWAS) of natural populations involving high-density single nucleotide polymorphisms (SNPs) detected by next-generation sequencing was used to dissect the genetic architecture of blast resistance (Kang et al. 2016) and bacterial blight resistance (Zhang et al. 2017) in rice. There were only two reports about the identification of ShB resistance QTLs by GWAS (Jia et al. 2012; Chen et al. 2019). A total of 10 ShB resistance QTLs were identified by an association mapping study involving 217 sub-core entries from the United States Department of Agriculture rice core collection and 155 simple sequence repeat markers (Jia et al. 2012). Recently, at least 11 SNP loci significantly associated with SB resistance at the seedling stage under artificial inoculation were detected by GWAS using 299 diverse rice varieties from the rice diversity panel with genotyping by 44,000 SNP markers array (Chen et al. 2019). However, no association studies have evaluated the diverse rice germplasm to identify potentially novel ShB resistance loci at the tillering stage based on genome-wide high-density SNPs. As part of the 3000 Rice Genomes Project (3K RGP) (3K RGP 2014), researchers recently used Illumina next-generation technology to sequence a core collection of 3024 rice accessions from 89 countries. Consequently, sequence data with a high coverage (approximately $94 \%$ ) and mapping rate (approximately 92.5\%) were generated for the construction of a high-density SNP database (Alexandrov et al. 2015), thereby providing genotype data for a GWAS of rice agronomic traits. Considerable genetic diversity regarding SNPs, structural variations, and gene presence/absence variations has been revealed among these cultivated rice accessions (Wang et al. 2018).

In the present study, 563 rice accessions, mainly belonging to Xian (also known as Indica), Geng (also known as Japonica), and Aus subgroups, from 47 countries and areas with similar heading dates as those in Beijing (China) were selected from the 3024 rice genomes sequenced by 3K RGP (3K RGP 2014; Wang et al. 2018). These accessions underwent an association analysis of their resistance to a representative $R$. solani strain from China. We used 2,977,750 SNPs filtered from the $3 \mathrm{~K}$ RGP 4.8 mio SNP dataset in the Rice SNP-Seek Database (Alexandrov et al. 2015). The objectives of our study were as follows: (1) identify ShB resistance resources in rice germplasm; (2) identify loci and candidate genes related to $\mathrm{ShB}$ resistance; and (3) elucidate the genetic mechanism underlying the quantitative resistance to $\mathrm{ShB}$ in rice. The data presented herein may be useful for improving ShB resistance by markerassisted selection in rice breeding programmes.

\section{Results}

Population Structure of Rice Accessions

A total of 220,335 independent SNPs with minor allele frequency $(\mathrm{MAF})>5 \%$ and missing data ratio $(\mathrm{MDR})<0.1$ were used for genetic structure analyses. A neighbourjoining tree developed using PHYLIP (version 3.6) (Felsenstein 1989), with 100 bootstrap replicates, revealed that the 563 accessions could be classified into three main clades (Fig. 1a). A population structure analysis using ADMIXTURE (Alexander et al. 2009) (optimal number of subpopulations $\mathrm{K}=3$ ) also indicated that the 563 accessions belonged to three distinct clusters (Fig. 1c). Similar results were observed for the principal component analysis with $67.02 \%$ of the genetic variation in the accessions explained by the first three principal components. When we plotted the first three components against each other, most accessions were clustered in three groups (Fig. 1b). Thus, the 563 rice accessions were classified into the following three well-known subgroups: Xian (224 accessions), Geng (237 accessions), and Aus (102 accessions) (Additional file 1: Table S1). These results suggested that the accessions used as a covariate within the GWAS model in this study exhibited an obvious subpopulation structure.

\section{Evaluation of the Resistance of Rice to $R$. solani}

An assessment of the resistance of the 563 tested accessions to $R$. solani based on the relative lesion height (RLH) revealed considerable variations within and between subgroups (Fig. 2). Moreover, 39 accessions with an RLH of 0.21-0.30 were considered to exhibit moderate resistance to $R$. solani based on the Standard Evaluation for rice (IRRI 2002). These accessions corresponded to 22 Aus and 17 Xian accessions (Additional file 1: Table S1). A dot plot indicated the lesion height (LH) was positively correlated with culm length (CL), but the correlation in Aus was much stronger than that in Geng, suggesting a strong differentiation in the resistance to $R$. solani among the three rice subgroups (Fig. 2a). The results of an analysis of variance demonstrated that the mean RLH and CL were 


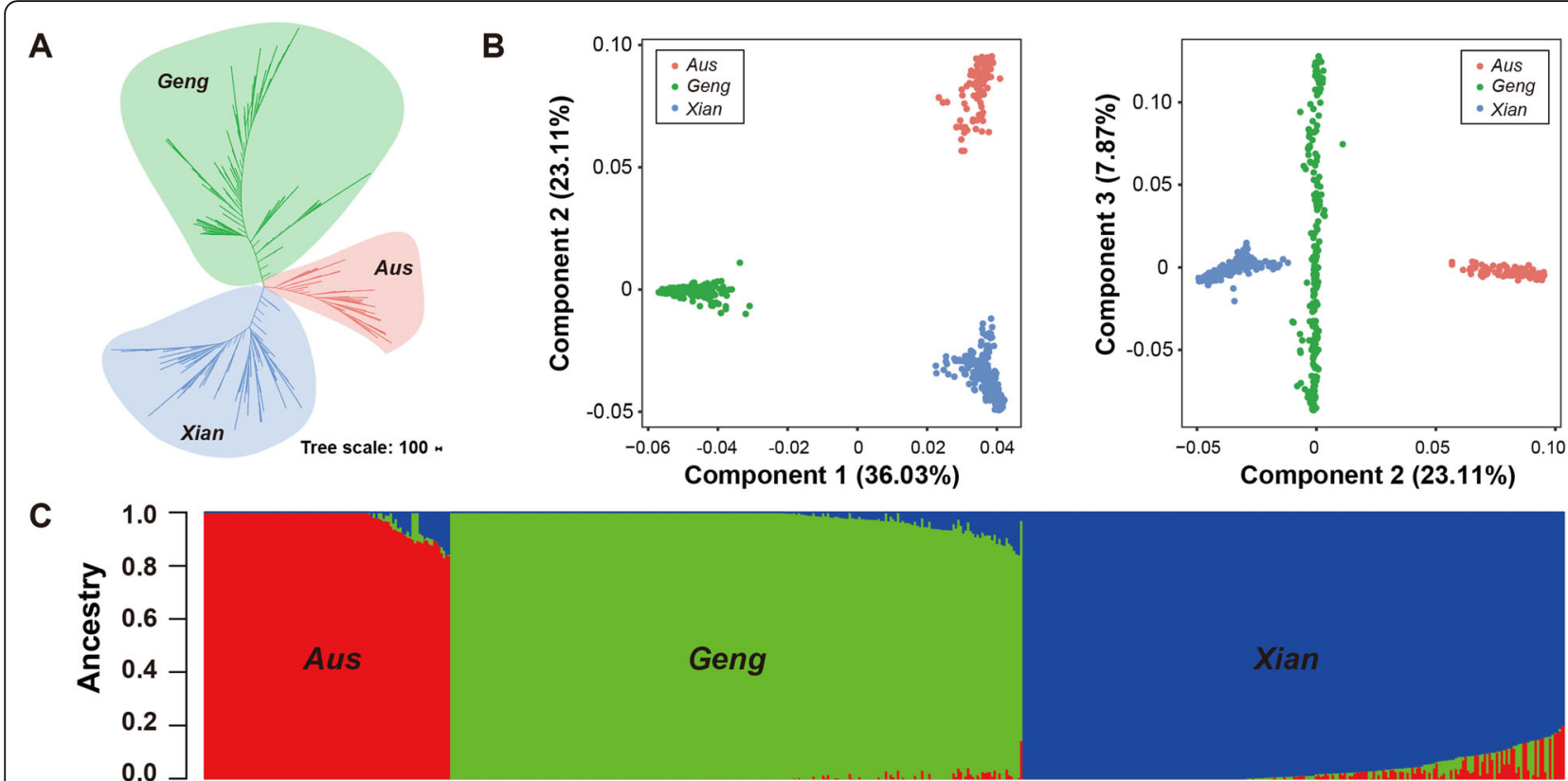

Fig. 1 Population structure of 563 rice accessions. a Neighbour-joining tree constructed from LD-pruned SNPs. b Principal component analysis plots for the first three components. Left: first and second components; right: second and third components. c Distribution of the estimated subpopulation components (ancestry fraction) for each accession as determined by ADMIXTURE

significantly lower and higher in the Aus subgroup than in the Geng and Xian subgroups, respectively. Additionally, the mean LH in the Xian subgroup was significantly lower than that in the Aus and Geng subgroups (Additional files 2 and 3: Tables S2 and S3). The average RLH values for the Aus, Xian, and Geng subgroups were 0.46, 0.52, and 0.61, respectively. Multiple comparisons revealed that the lowest RLH value was associated with the Aus subgroup (Fig. 2b), implying that the Aus accessions were more resistant to ShB than the accessions in the other two subgroups. The results of a correlation analysis among CL, LH, and RLH values across the whole panel and three subgroups are presented in Fig. 2c. There were significant positive correlations between LH and RLH in the whole panel and three subgroups, with the strongest correlation $(r=0.92$, $P<0.01$ ) detected in the Aus subgroup (Fig. 2c).
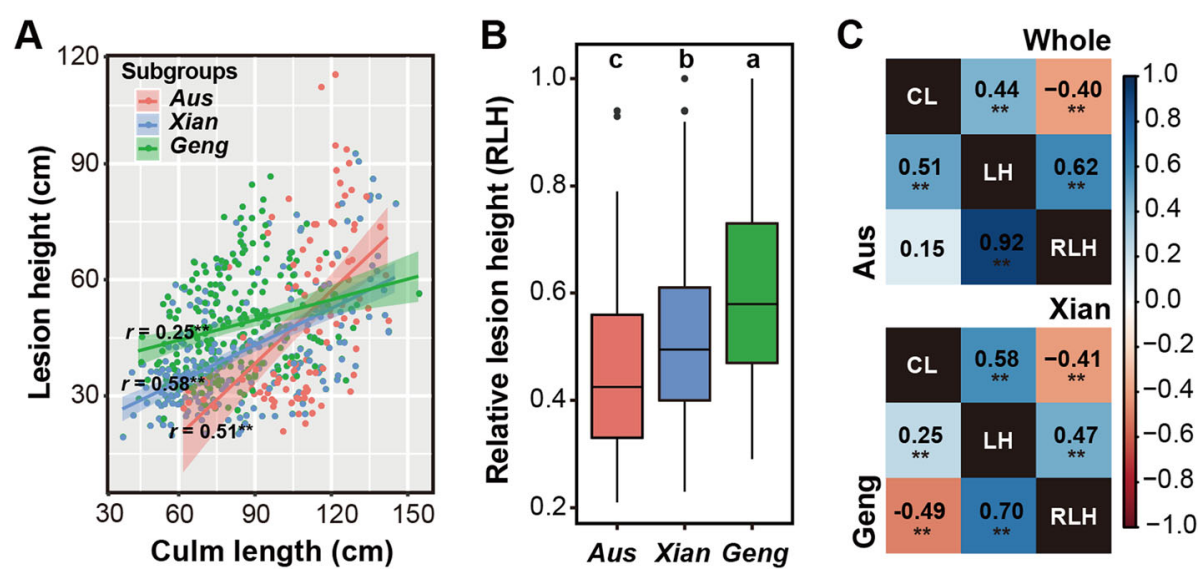

Fig. 2 Evaluation of the traits related to ShB resistance and correlations among traits observed in different rice subgroups. a Dot plot of correlations between culm length $(\mathrm{CL})$ and lesion height (LH). Orange, blue, and green dots represent Aus, Xian, and Geng accessions, respectively. Orange, blue, and green trend lines and shadowing represent linear regression lines and confidence intervals, respectively, between $\mathrm{CL}$ and LH within Aus, Xian, and Geng subgroups. b Relative lesion height (RLH) in different subgroups. Different characters indicate significant differences between rice subgroups $(P<0.01)$. c Correlations among $C L, L H$, and RLH from each GWAS panel. The number in the middle of the cell is the correlation coefficient and ${ }^{* * \prime}$ refers to a significant correlation $(P<0.01)$ 
Interestingly, there were significant negative correlations between CL and RLH in the whole panel as well as the Xian and Geng subgroups, but not in the Aus subgroup (Fig. 2c).

\section{Genome-Wide Association Signals for Sheath Blight Resistance in Rice}

We conducted association studies to identify genomewide associated signals underlying the quantitative resistance to R. solani in the whole, Aus, Xian, and Geng panels to minimize the impact of rice population structure on the detection power of the GWAS. A total of 2, 977,750 (whole), 1,665,543 (Aus), 1,776,496 (Xian), and $1,314,743$ (Geng) SNPs with MAF $>5 \%$ and MDR $<0.1$ were used for the association analyses according to the single-locus linear mixed model (LMM) by the Efficient Mixed Model Association eXpedited (EMMAX) and the multi-locus LMM by a fixed and random model with a circulating probability unification (FarmCPU) (Additional files 10, 11, 12, 13, 14: Figures S1-S5 and Fig. 3). Additionally, on the basis of a Bonferroni correction involving the effective number of independent markers at a significance level of 0.05 (Li et al. 2012), the genome-wide suggestive thresholds were $P=2.16 \times 10^{-6}$, $3.28 \times 10^{-6}, 6.57 \times 10^{-6}$, and $5.34 \times 10^{-6}$ for the whole, Xian, Geng, and Aus panels, respectively (Additional file 4: Table S4). A total of 562 and 209 suggestive association signals were detected in one or more of the panel populations by EMMAX and FarmCPU, respectively (Additional file 5: Table S5). We identified 676 suggestively associated SNPs with different physical positions, namely quantitative trait nucleotides (QTNs), in at least one of the GWAS panels by EMMAX or FarmCPU, including 132, 498, and 62 QTNs for CL, LH, and RLH, respectively (Additional file 5: Table S5). Of the 676 QTNs, 553 SNPs either produced a synonymous substitution or were located in intergenic regions and introns. According to the reference Nipponbare genome IRGSP 1.0, the remaining SNPs were associated with large effects, and were detected in promoters (57), missense variants (32), 5' UTRs (9), 3' UTRs (14), start codons (1), splice regions (4), and stop codons (6) (Additional file 5: Table S5). Details regarding these suggestive association signals are listed in Additional file 5: Table S5. The average physical distance between neighbouring SNPs was 125.2, 223.8, 209.9, and $283.5 \mathrm{bp}$ in the whole, Aus, Xian, and Geng panels, respectively. The average estimated linkage disequilibrium (LD) block region for all 12 chromosomes in the whole panel was $20.6 \mathrm{~kb}$, ranging from $12.1 \mathrm{~kb}$ on chromosome 11 to $50.7 \mathrm{~kb}$ on chromosome 3 . Thus, we combined adjacent QTNs within an LD block to form a suggestively associated locus (SAL) for ShB resistance in each GWAS panel. Moreover, the QTN with the minimum $P$ value in a cluster was considered to be the lead
SNP. We focused on the identified RLH associations due to the major parameter used to assess the ShB resistance in this study (Fig. 3 and Additional file 14: Figure S5).

A total of 27 SALs for ShB resistance containing 62 suggestive QTNs (including 11 SALs containing 12 significant QTNs) were detected on all chromosomes except for chromosome 8 by merging the QTNs based on EMMAX or FarmCPU in all panels (Table 1). Remarkably, significant QTNs for RLH were not identified in the Aus and Xian panels, and no QTN was detected in more than one GWAS panel. At least 12 SALs (L4, L7, L10, L13, L15, L16, L18, L19, L23, L25, L26, and L27) were adjacent to one or more previously characterized ShB resistance-related QTLs/genes. In the whole panel, L3 (17.85-17.96 Mb) on chromosome 2 and L15 (15.24-15.25 Mb) on chromosome 7, had the most significant association signals for RLH. We also determined that another previously reported ShB resistance QTL (qSBR7-1) in Tetep (Channamallikarjuna et al. 2010) was located nearby the L15 region. Notably, no QTL/ gene related to $\mathrm{ShB}$ resistance was reported in the L3 region (17.85-17.96 Mb) on chromosome 2, which included the most significant association signal (rs2_17, $889,517, P=4.8 \times 10^{-14}$ ) in the whole panel, suggesting this region may contain a potentially novel SAL that should be fine-mapped (Table 1). In the Geng panel, L23 $(14.42-14.59 \mathrm{Mb})$ on chromosome 10 and L25 (5.70$5.77 \mathrm{Mb}$ ) on chromosome 11 were the only two SALs related to significant association signals. In the Aus panel, there were two SALs localized to two hotspot regions, namely L4 on chromosome 2 and L26 on chromosome 11, detected for RLH and LH by EMMAX and FarmCPU. The lead SNP (rs11_25,580,510, $P=7.0 \times 10^{-7}$ ) of L26 was located in the intergenic region between $L O C_{-}$ Os11g42450 and LOC_Os11g42470, which encode an LRR family protein and a protein with an unknown function, respectively. In the Xian panel, only SAL L5 (16.20-16.25 Mb) on chromosome 3 was detected. Similar to L3, L5 was not associated with previously reported ShB resistance QTLs/genes. The lead SNP (rs3_16,214, 232, $P=9.5 \times 10^{-7}$ ) of L5 was detected in the promoter region of a gene associated with an unknown function (LOC_Os03g28170).

\section{Identifying Putative Functional Genes Associated with Sheath Blight Resistance}

For a given GWAS locus, the gene nearest to the lead SNP is not always the causal gene (Brodie et al. 2016). Therefore, all genes located in the LD blocks of detected SALs underwent an extensive haplotype analysis to identify putative functional genes. The LD blocks of all detected SALs except for L1 and L27 included more than one gene annotated based on the Nipponbare reference genome IRGSP 1.0. A total of 316 genes were detected 


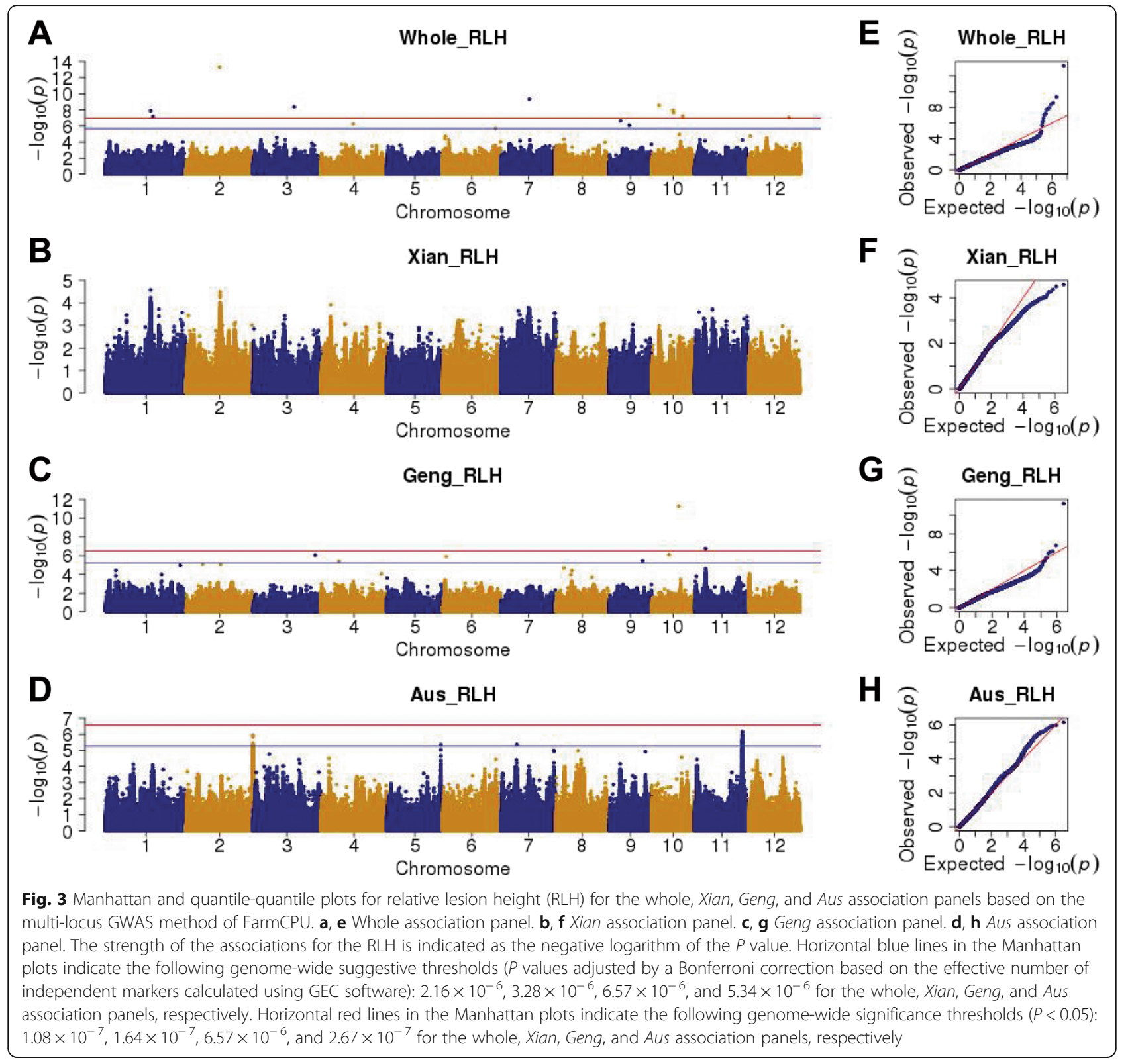

in 25 LD blocks (L2-L26). Of these genes, 283 with at least one SNP in the whole GWAS panel were included in a haplotype analysis (e.g., haplotype frequency in each subpopulation) and multiple comparison tests of the RLH in each GWAS panel. Consequently, 261 ShB resistance putative functional genes (SRPFGs) were identified in all SALs except for L1, L12, L13, and L27, with significant differences in the RLH among different haplotypes in at least one GWAS panel. These 261 genes comprised 116 functionally annotated genes, 57 transposons, and 88 genes with unknown functions (Additional file 6: Table S6). Our findings may be useful for identifying the genes responsible for $\mathrm{ShB}$ resistance. The number of SRPFGs per SAL ranged from 1 in L22 to 25 in L23, with a mean of $11.3 \pm 7.3$. These genes were significantly enriched in the gene ontology (GO) biological processes related to plant cellular amino acid metabolic processes (Additional file 7: Table S7). Moreover, they were also significantly enriched in plant metabolic pathways, including fatty acid biosynthesis and degradation based on the Kyoto Encyclopedia of Genes and Genomes (KEGG) pathway database (Additional file 8: Table S8). Out of the 204 non-transposon SRPFGs, we detail showed haplotype analyses of 36 genes within 20 detected SALs (Additional file 9: Table S9) if it met any of the following conditions: (i) involving in GO classification of response to stress term; (ii) genes with known function on disease resistance; (iii) as one hit gene in the 


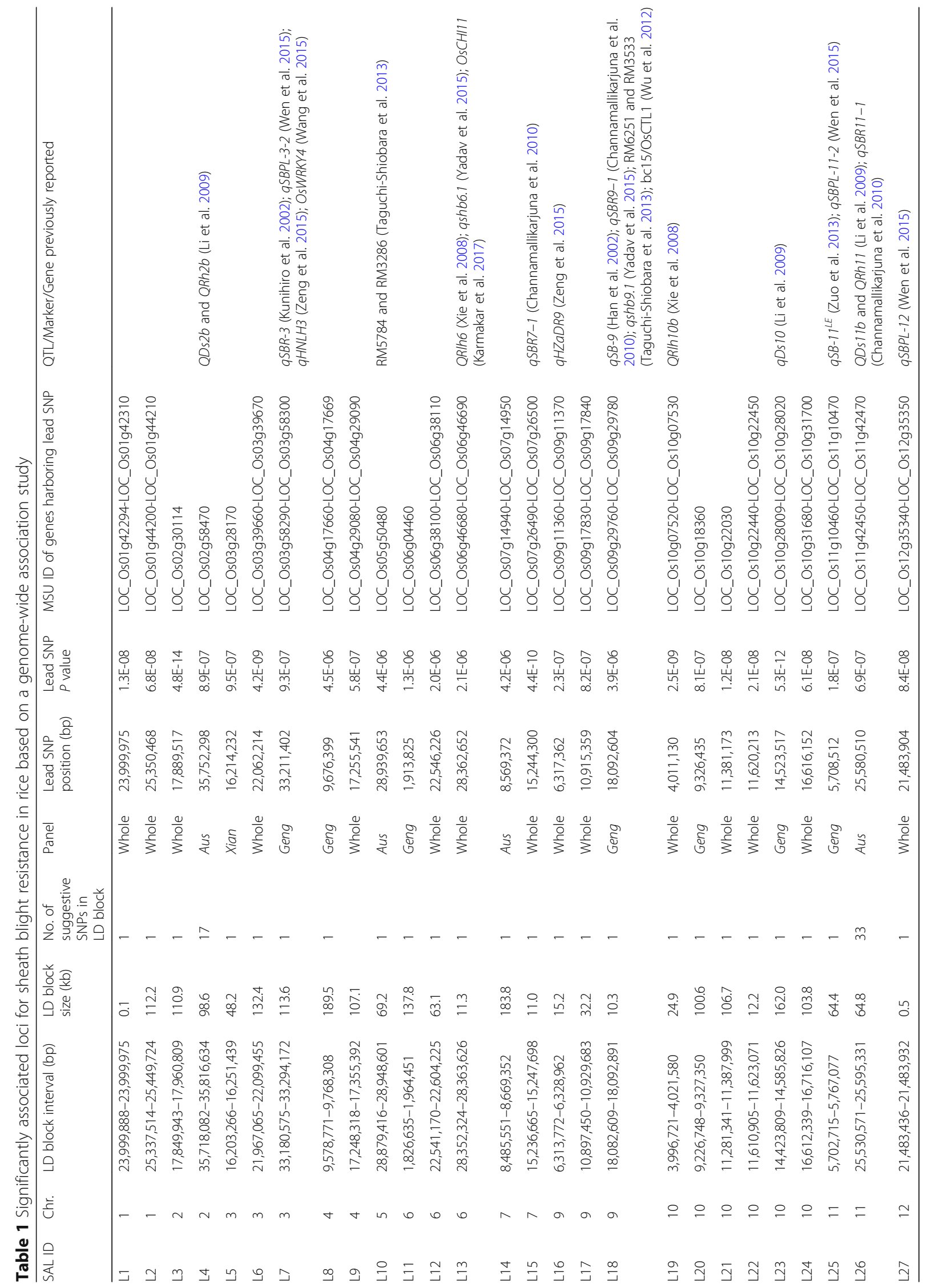


significantly enriched pathways, and (iv) the relatively lower $p$-value of the haplotype analyses in Additional file 6: Table S6. Two of these genes (LOC_Os10g28050 and LOC_Os06g04510) were subsequently analysed as follows.

We detected LOC_Os10g28050, which encodes a chitinase 2, in the LD block region of L23. This gene was located $34 \mathrm{~kb}$ downstream of the lead SNP rs10_14,523, 517 (Fig. 4a). The haplotypes were built based on six SNPs in a $1-\mathrm{kb}$ region upstream of the $L O C_{-}$ Os10g28050 promoter, one SNP in the coding region with a missense mutation, and two SNPs in the 3' UTR (Fig. 4b). We detected five haplotypes shared by at least 10 accessions in 501 of 563 accessions (Fig. 4b and Additional file 9: Table S9). A comparison of the RLHs for the five haplotypes revealed that accessions Hap2 and Hap5 had significantly lower RLHs than the other three haplotypes $(P<0.01$; Fig. $4 \mathrm{c}$ and Additional file 9: Table S9). Furthermore, in the Aus panel, Hap2 was represented by $70(74.5 \%)$ of 94 accessions, while $14(82.4 \%)$ of 17 accessions corresponded to Hap5. In contrast, the Geng panel lacked Hap2 and Hap5 accessions (Fig. 4c and Additional file 9: Table S9). These results partially explained the variability in the resistance to $R$. solani observed among the Aus, Xian, and Geng panels.

We located LOC_Os06g04510, which encodes an enolase, in the LD block region of L11. This gene was located $17 \mathrm{~kb}$ downstream of the lead SNP rs6_1,913,825 (Fig. 4d). According to the Nipponbare reference genome IRGSP 1.0, LOC_OsO6g04510 is associated with two transcripts. We analysed these two transcripts, and identified four major haplotypes shared by at least 10 of 479 accessions based on eight SNPs in a $1-\mathrm{kb}$ upstream region, gene coding region, and the $5^{\prime}$ and $3^{\prime}$ UTRs (Fig. 4e). We compared the mean RLH among those haplotypes in each GWAS panel. The RLH of Hap1 was significantly higher than that of the other three haplotypes in the whole panel. Notably, in the Geng panel, the RLH of Hap4 was significantly lower than that of Hap1 $(P=0.0045)$ (Fig. 4f and Additional file 9: Table S9). Among the 224 accessions with the Hap1 major haplotype, $196(87.5 \%)$ accessions belonged to the Geng panel. Additionally, all but one of the Hap2 accessions were from the Xian and Aus panels, with the exception belonging to the Geng panel. Of the two minor haplotypes, 91.7\% of Hap3 accessions belonged to the Aus panel, while 93.8\% of Hap4 accessions belonged to the Geng panel. These results were useful for clarifying the phenotypic variability within the Geng panel in response to $R$. solani.

\section{Discussion}

During the past 3 decades, considerable efforts have been made to screen rice germplasm for ShB resistance (Srinivasachary and Savary 2011; Yadav et al. 2015).
However, no cultivars with complete resistance are available and no major genes conferring immunity to $R$. solani have been identified. Most QTLs conferring ShB resistance identified using bi-parental linkage mapping populations exhibited small effects (Zeng et al. 2011). A GWAS based on diversified germplasm with highdensity genotypes may provide new insights into the genetic basis of agronomic traits in crops. In the present study, we conducted a large-scale GWAS based on a rice core collection and approximately 3 million SNPs to dissect the genetic mechanisms underlying quantitative resistance to $R$. solani and to identify novel loci and alleles associated with resistance. Our results may provide useful information for improving ShB resistance in rice.

\section{Diverse Reactions of Accessions to Rhizoctonia solani among Rice Subgroups}

In this study, multiple comparisons of RLH among three panels revealed Aus accessions were the most resistant to $\mathrm{ShB}$, followed by the Xian and Geng accessions (Fig. 2b), which is consistent with the results of previous studies (Chen et al. 2019; Willocquet et al. 2012; Zuo et al. 2008). The data from bi-parental mapping populations in earlier investigations implied that RLH is negatively correlated with plant height or $\mathrm{CL}(\mathrm{Li}$ et al. 2009; Li et al. 1995; Wen et al. 2015), and seedling height and disease score of $\mathrm{ShB}$ resistance is aslo negatively correlated in rice diversity panel 1 (Chen et al. 2019). Our results also showed significantly negative correlations between RLH and CL in Xian and Geng accessions (Fig. 2c). Therefore, CL significantly affected the evaluation of the ShB resistance based on RLH. However, there was a non-significant correlation between RLH and CL in the Aus accessions (Fig. 2c), indicating that the influence of $\mathrm{CL}$ on evaluation of $\mathrm{ShB}$ resistance can be effectively eliminated in Aus panel. One possible reason for the differing relationship between CL and RLH in Aus compared with Xian and Geng is the relatively large variation of $\mathrm{LH}$ versus small of variation of $\mathrm{CL}$ in Aus (Additional file 4: Table S3), which explains the Type II SALs L4 and L26 could be detected for both RLH and LH (Additional file 5: Table S5). We further examined the relationships between the RLH- and CL-SALs. Only one co-localization of CL-SAL and RLH-SAL L5 at approximately $16.2 \mathrm{Mb}$ on chromosome 3 was detected in the Xian panel (Additional file 5: Table S5). These results suggested that most of the RLH-SALs identified in this study were more likely to control the molecular mechanisms underlying physiological resistance mentioned by Srinivasachary et al. (Srinivasachary and Savary 2011) rather than the disease escape strongly determined by plant architecture. These findings may be useful for enhancing ShB resistance in rice cultivars. 


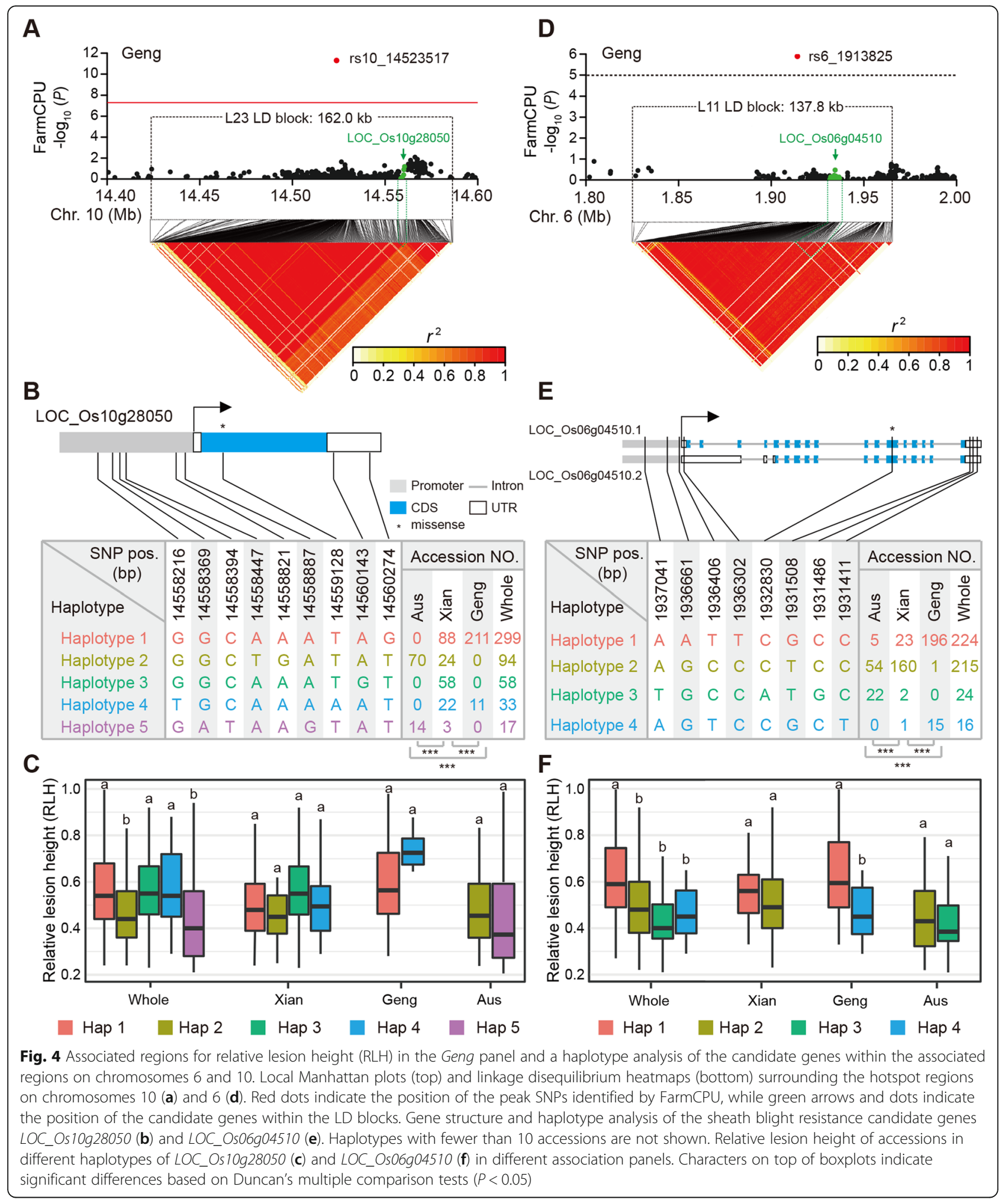

\section{Comparison of SAL and QTL Mapping for Sheath Blight Resistance}

According to our results, association studies involving a combination of a single locus genome-wide scan
(EMMAX) and a multi-locus GWAS method (FarmCPU) are more efficient in determining the number of loci controlling rice quantitative resistance to $R$. solani than classical bi-parental linkage mapping methods. One 
possible explanation is that only QTLs with segregating alleles between limited founder parents can be identified by traditional QTL linkage mapping. We observed that $12(44.4 \%)$ of 27 GWAS loci associated with RLH harboured multiple previously reported QTLs/genes related to ShB resistance (Table 1). For example, in the chromosomal region near SAL L25, one well-known major QTL $\left(q S B-11^{\mathrm{LE}}\right)$ for $\mathrm{ShB}$ resistance was fine-mapped using a set of chromosome segment substitution lines in the Lemont genetic background with Teqing as a donor parent (Zuo et al. 2013). Meanwhile, another QTL (qSBPL-11-2) was detected using three Lemont/Yangdao4 mapping populations (Wen et al. 2015) (Table 1 and Additional file 5: Table S5). Additionally, SAL L23, with the most significant SNP (rs10_14,523,517, $P=5.3 \times 10^{-12}$ ) associated with RLH in the Geng panel, contained one QTL ( $q D s 10)$ for disease severity in a Teqing/Binam backcross introgression line (BIL) population (Li et al. 2009) (Table 1 and Additional file 5: Table S5). Moreover, two previously reported QTLs (QDs2b and QRh2b) (Li et al. 2009) identified in a Teqing/Tarom Molaii BIL population were found adjacent to the L4 region $(35.72-35.82 \mathrm{Mb})$. Moreover, SAL L26 included 33 suggestively associated SNPs spanning an approximately 64.8 -kb interval $(25.53-25.60 \mathrm{Mb})$ on chromosome 11, with another two reported ShB resistance QTLs (QDs11b and QRh11) from a Teqing/Tarom Molaii BIL population ( $\mathrm{Li}$ et al. 2009) and one finemapped QTL (qSBR11-1) from rice line Tetep with a high degree of resistance to $R$. solani (Channamallikarjuna et al. 2010) (Table 1 and Additional file 5: Table S5). The co-localization of known QTLs/genes helped to verify the SALs identified in our study. These confirmed SALs represent candidates for fine-mapping, gene cloning, and marker-assisted selection for improving the ShB resistance of rice cultivars.

Putative Defence Mechanism against Sheath Blight in Rice The molecular mechanisms involved in the host-pathogen interactions underlying quantitative $\mathrm{ShB}$ resistance in rice remain unclear. Some studies concluded that a jasmonic acid (JA)-induced resistance pathway (Karmakar et al. 2017; Wang et al. 2015) and a salicylic acid (SA)-mediated systemic acquired resistance pathway (Kouzai et al. 2018; Molla et al. 2016) might influence the resistance of rice to $R$. solani. These two important pathways, which form part of the defence system in rice, have common defencerelated and pathogenesis-related proteins such as chitinases (Datta et al. 2001; Karmakar et al. 2016; Karmakar et al. 2017; Richa et al. 2017), glucanases (Datta et al. 1999), and OsWRKY transcription factors (Wang et al. 2015). Chitinase production can be induced by JA in rice (Rakwal et al. 2004). One chitinase gene, LOC_Os11g47510, was recently cloned from a QTL region (qSBR11-1) for ShB resistance in the $R$. solani-tolerant rice line Tetep, and subsequently validated by a genetic transformation into susceptible rice line Taipei 309 (Richa et al. 2017). The overexpression of several other chitinase genes, such as OsCHI11 and RCH1O, have resulted in increased $\mathrm{ShB}$ tolerance in transgenic plants (Lin et al. 1995; Mao et al. 2014).

The involvement of SA, which is a product of phenylpropanoid metabolism (Lee et al. 1995), in the resistance of rice to $R$. solani was confirmed in SA-deficient transgenic plants (Kouzai et al. 2018). The glycolytic pathway is reportedly important for defence responses against $R$. solani and is connected to the phenylpropanoid pathway (Mutuku and Nose 2012). Additionally, SA biosynthesis may be indirectly induced by cuticular wax accumulation during resistance responses in plants (Kouzai et al. 2018; Seo et al. 2011). Cuticular wax is mainly composed of long-chain aliphatic compounds, and wax biosynthesis in plants begins with the synthesis of fatty acids in the plastid (Kunst and Samuels 2003). The wax forms a natural barrier against biotic and abiotic stresses during plant growth and development (Wang et al. 2017). In rice cultivars, the amount of cuticular wax deposits on the outer sheaths are negatively correlated with the infection rates and formation of infection cushions by $R$. solani (Marshall and Rush 1980).

In the present study, $36 \mathrm{ShB}$ resistance genes were screened from 261 SRPFGs within LD blocks of 23 RLH-SALs based on the annotation of gene functions, enriched KEGG pathways and GO terms, and multiple comparisons among haplotypes (Additional file 9: Table S9). Our data suggest that JA and SA signalling pathways might regulate rice responses to ShB. For example, LOC_Os10g28050, which encodes chitinase 2, was identified $34 \mathrm{~kb}$ downstream of the lead SNP rs10_14,523, 517 of the L23 LD block (Fig. 4a). The mean RLHs of the Hap2 and Hap5 accessions carrying LOC_ Os10g28050 were significantly lower than that of the other three haplotypes. The resistance haplotypes (Hap2 and Hap5) were mainly enriched in the Aus panel, and the five haplotypes also exhibited a distribution tendency among subgroups (Fig. 4c and Additional file 9: Table S9). Additionally, LOC_Os06g04510, which encodes an enolase that participates in the glycolytic pathway, was detected $17 \mathrm{~kb}$ downstream of the lead SNP rs6_1,913, 825 of SAL L11 (Fig. 4d). The mean RLH of Hap3 accessions with this gene was significantly lower than that of the other three haplotypes. Similar to LOC_Os10g28050, the LOC_Os06g04510 resistance haplotype of Hap3 accessions was mainly enriched in the Aus panel. Furthermore, two haplotypes (Hap1 and Hap4) explained the phenotypic variability within the Geng panels in response to $R$. solani (Fig. 4c and Additional file 9: Table S9).

In this study, significant differences in the mean RLH among different haplotypes within subgroups or the whole population were identified for two genes ( $L O C_{-}$ 
OsO2g30060 and LOC_Os10g31780) involved in fatty acid biosynthesis and one gene (LOC_Os11g10520 encoding a dehydrogenase) related to the degradation of aromatic compounds (Additional files 8 and 9: Tables S8 and S9). Molecular studies have revealed that rice plants expressing the gene encoding OsGL1-1, which contains regions homologous to parts of short-chain dehydrogenases, exhibit induced deposition of cuticular wax in contrast to the osgl1-1 mutant lacking the corresponding gene (Qin et al. 2011).

Transient expression assays revealed that SA-inducible OsWRKY6 is a positive regulator of a constitutively activated pathogenesis-related gene (OsPR10a), and OsWRKY6-overexpressing transgenic rice plants exhibit enhanced resistance to pathogens (Choi et al. 2015). In this study, we determined that OsWRKY6 (LOC_OsO3g58420), located in SAL L7 near three previously mapped QTL regions ( $q S B R-3, q S B P L-3-2$, and $q H N L H 3)$ is associated with ShB resistance (Additional file 9: Table S9). Regarding this gene, multiple comparisons of the RLH revealed that Hap3 accessions were most resistant to ShB, followed by Hap2 and Hap1 accessions. Moreover, Hap3 was absent in the Xian and Geng accessions, but was relatively abundant in the Aus panel, confirming a strong differentiation among three rice subgroups. However, some OsWRKY transcription factors, such as OsWRKY4 and OsWRKY30, are reportedly important positive regulators of rice responses to ShB mediated by a JA-dependent signalling pathway (Peng et al. 2012; Wang et al. 2015). These results provide novel information regarding the genes involved in ShB resistance and further elucidate the molecular mechanism underlying rice resistance to $\mathrm{ShB}$.

\section{Conclusions}

The current GWAS of ShB resistance detected suggestive signals, estimated the candidate regions with suggestive signals based on LD blocks, and predicted the causal genes according to bioinformatics and haplotype analyses. Our results imply that quantitative resistance to $R$. solan $i$ in rice may be mediated by SA- and JA-dependent signalling pathways. Our future research will focus on the functional validation of the identified candidate genes by genetic transformations and transcriptomics-based investigations. The findings reported herein may be useful for improving the application of rice germplasm resources as well as the knowledge-based management of ShB and the breeding of ShB-resistant rice cultivars.

\section{Methods}

Rice Germplasm and Evaluation of Sheath Blight Resistance under Field Conditions

We selected a diverse collection of 563 Oryza sativa accessions from 47 countries and areas without distinct unfavourable agronomic traits and with similar heading dates as those in Beijing, China $\left(116^{\circ} 20^{\prime} \mathrm{E}, 40^{\circ} 22^{\prime} \mathrm{N}\right)$ from the 3K RGP database (3K RGP 2014) (Additional file 1: Table S1). To evaluate ShB resistance, the seeds of all rice accessions were sown in a seedling nursery, and 30-day-old seedlings were transplanted to the experimental farm at the Institute of Crop Sciences, Chinese Academy of Agricultural Sciences, Beijing, China. Each row $(20 \times 17 \mathrm{~cm})$ comprised nine plants, which were inoculated with the highly pathogenic $R$. solani strain RH9 at the late tillering stage as described by Zou et al. (Zou et al. 2000). Three central plants for each line were inoculated (with two replicates), with the third leaf sheath of the main stem and the four largest tillers of each plant inserted into the inoculum without changing the holding status of the sheath and stem (Zuo et al. 2008). The ShB resistance of each inoculated plant was evaluated 30 days later (Zeng et al. 2015) according to the RLH, which was calculated as the ratio between LH and CL. The three tillers with the highest lesions were selected for each plant, and the LH was measured along the stem from the lowest to highest sites, while the CL was estimated from the soil surface to the panicle neck. The mean trait value of one accession was calculated based on three individual plants (i.e., three lesions per plant) for each replicate. The average trait values of two replicates for each accession were used for the GWAS.

\section{Population Structure Analysis}

The 3K RGP 4.8 mio SNP dataset was downloaded from the Rice SNP-Seek Database. http://snp-seek.irri.org/ (Alexandrov et al. 2015). To avoid the influence of linked SNPs during the population structure analysis, we used the LD pruning tool of the PLINK program (version 1.9) (Purcell et al. 2007) to obtain a subset of 220, 335 independent SNPs with a MAF $>5 \%$ and a MDR < 0.1 according to 'indep-pairwise 50100.5 '. We used PHYLIP (version 3.6) (Felsenstein 1989) to construct an unrooted neighbour-joining tree with 100 bootstrap replicates. The genetic structure of the whole population was predicted with the ADMIXTURE program (Alexander et al. 2009). Meanwhile, PLINK (Purcell et al. 2007) was used to conduct a principal component analysis to estimate the number of subpopulations in the GWAS panel.

\section{Genome-Wide Association Mapping}

A total of 2,977,750 SNPs with a MAF $>5 \%$ and MDR < 0.1 were filtered for association analyses of the whole panel. The GWAS was completed using a LMM implemented in EMMAX program (Kang et al. 2010) as well as FarmCPU (Liu et al. 2016) to determine the associations between each SNP and three traits related to ShB resistance ( $\mathrm{LH}, \mathrm{CL}$, and $\mathrm{RLH})$. We used the BaldingNichols matrix based on a pruned subset of 65,095 SNPs 
across the whole rice genome (with parameter indeppairwise 50100.1 ' in PLINK) to develop the kinship matrix, which measured the genetic similarity between individuals. The first three principal components were used as covariates (Q-matrix) to control for population structure. The effective number of independent markers (N) was calculated using GEC software (Li et al. 2012), and the suggestive and significant $P$-value thresholds of each GWAS panel were calculated (Additional file 4: Table S4). The Manhattan and quantile-quantile plots for the GWAS results were created using the R package qqman (Turner 2014). To obtain independent association signals, multiple suggestively associated SNPs located in one estimated LD block were clustered as one SAL, and the SNP with the minimum $P$ value in a cluster was considered as the lead SNP. The LD block was estimated with the command '--blocks' in PLINK according to the block definition suggested by Gabriel et al. (Gabriel et al. 2002). The pairwise LD $r^{2}$ values within one estimated LD block were calculated with PLINK (Purcell et al. 2007), and the R package LDheatmap (Shin et al. 2006) was used to draw the heatmap of pairwise LDs.

\section{Annotation of Significant Signals}

Synonymous and nonsynonymous SNPs and SNPs associated with large-effect changes were annotated based on the gene models of the annotated version of the Nipponbare reference genome IRGSP 1.0 (Kawahara et al. 2013) using the snpEff program (version 4.0) (Cingolani et al. 2012). Enriched GO terms and KEGG pathways were identified using the agriGO v2.0 (Tian et al. 2017) and EXPath 2.0 (Chien et al. 2015) programs, respectively. We have listed all suggestively associated SNPs located within genes and the annotation information based on the Nipponbare reference genome IRGSP 1.0 (Kawahara et al. 2013).

\section{Haplotype Analysis}

The whole GWAS panel SNPs within $1 \mathrm{~kb}$ of the upstream promoter region, 3' untranslated region (UTR), and $5^{\prime}$ UTR as well as non-synonymous SNPs in the coding regions of a candidate gene were concatenated as the haplotype. Only haplotypes shared by at least 10 accessions were used for multiple comparisons. For the multiple group comparison of the RLHs of the major haplotypes, Duncan's multiple comparison tests followed by a one-way analysis of variance were completed with the agricolae package in R. Additionally, chi-square tests in $\mathrm{R}$ were used to determine significant differences in the frequency of different haplotypes for the candidate genes among rice subgroups.

\section{Supplementary information}

Supplementary information accompanies this paper at https://doi.org/10. 1186/s12284-019-0351-5.

Additional file 1 : Table S1. Summary of 563 rice accessions and their resistance to rice sheath blight.

Additional file $\mathbf{2}$ : Table S2. Analysis of the variance in traits related to sheath blight resistance.

Additional file $\mathbf{3}$ : Table S3. Multiple comparisons of traits related to sheath blight resistance among three rice subgroups.

Additional file 4 : Table S4. Filtered and effective number of single nucleotide polymorphisms across panels and adjusted significant $P$ value thresholds based on a Bonferroni correction.

Additional file $\mathbf{5}$ : Table $\mathbf{S 5}$. Significant signals associated with resistance to Rhizoctonia solani detected in at least one of the rice panel populations based on two GWAS models.

Additional file $\mathbf{6}$ : Table S6. Sheath blight resistance putative functional genes for 23 RLH-SALs detected by a GWAS.

Additional file $\mathbf{7}$ : Table S7. Results of a GO enrichment analysis of sheath blight resistance putative functional genes.

Additional file 8 : Table S8. Results of a KEGG pathway enrichment analysis of sheath blight resistance putative functional genes.

Additional file 9 : Table S9. Haplotype analyses of 36 genes associated with RLH screened based on annotated functions and multiple comparisons.

Additional file 10 : Figure S1. Manhattan and quantile-quantile plots for culm length based on the whole, Xian, Geng, and Aus panels using EMMAX.

Additional file 11 : Figure S2. Manhattan and quantile-quantile plots for culm length based on the whole, Xian, Geng, and Aus panels using FarmCPU.

Additional file 12 : Figure S3. Manhattan and quantile-quantile plots for lesion height based on the whole, Xian, Geng, and Aus panels using EMMAX.

Additional file 13 : Figure S4. Manhattan and quantile-quantile plots for lesion height based on the whole, Xian, Geng, and Aus panels using FarmCPU.

Additional file 14 : Figure S5. Manhattan and quantile-quantile plots for relative lesion height based on the whole, Xian, Geng, and Aus panels using EMMAX

\section{Abbreviations}

3K RGP: 3,000 Rice Genomes Project; BIL: Backcross introgression line; CL: Culm length; EMMAX: Efficient Mixed Model Association eXpedited; FarmCPU: Fixed and random model with a circulating probability unification; GO: Gene ontology; GWAS: Genome-wide association study; JA: Jasmonic acid; KEGG: Kyoto Encyclopedia of Genes and Genomes; LD: Linkage disequilibrium; LH: Lesion height; LMM: Linear mixed model; MAF: Minor allele frequency; MDR: Missing data ratio; QTL: Quantitative trait locus; QTNs: Quantitative trait nucleotides; R: solani: Rhizoctonia solani Kühn; RLH: Relative lesion height; SA: Salicylic acid; SAL: Suggestively associated locus; ShB: Sheath blight; SNPs: Single nucleotide polymorphisms; SRPFGs: ShB resistance putative functional genes

\section{Acknowledgements}

We thank Liwen Bianji, Edanz Editing China (www.liwenbianji.cn/ac) for editing the English text of a draft of this manuscript.

\section{Authors' Contributions}

Conceived and designed the experiments: YZ; Performed the experiments: FZ DZ CZ JL TC JX; Analyzed the data: FZ; Wrote the paper: FZ YZ. All authors read and approved the final mansucript.

\section{Funding}

This research was supported by grants from the National Key Research and Development Program of China (Project No. 2016YFD0100101), the National 
High-tech Program of China (No. 2014AA10A603), the Bill \& Melinda Gates Foundation (OPP1130530) and the CAAS Agricultural Science and Technology Innovative Program.

\section{Availability of Data and Materials}

All data supporting the conclusions of this article are provided within the article (and its Additional files).

\section{Ethics Approval and Consent to Participate}

Not applicable.

\section{Consent for Publication}

Not applicable.

\section{Competing Interests}

The authors declare that they have no competing interests.

Received: 11 September 2019 Accepted: 25 November 2019 Published online: 18 December 2019

\section{References}

3K RGP (2014) The 3,000 rice genomes project. Gigascience 3:7

Alexander DH, Novembre J, Lange K (2009) Fast model-based estimation of ancestry in unrelated individuals. Genome Res 19:1655-1664

Alexandrov N, Tai S, Wang W, Mansueto L, Palis K, Fuentes RR, Ulat VJ, Chebotarov D, Zhang G, Li Z, Mauleon R, Hamilton RS, McNally KL (2015) SNP-seek database of SNPs derived from 3000 rice genomes. Nucleic Acids Res 43:D1023-D1027

Brodie A, Azaria JR, Ofran Y (2016) How far from the SNP may the causative genes be? Nucleic Acids Res 44:6046-6054

Channamallikarjuna V, Sonah H, Prasad M, Rao GJN, Chand S, Upreti HC, Singh NK, Sharma TR (2010) Identification of major quantitative trait loci qSBR11-1 for sheath blight resistance in rice. Mol Breed 25:155-166

Chen Y, Zhang AF, Wang WX, Zhang Y, Gao TC (2012) Baseline sensitivity and efficacy of thifluzamide in Rhizoctonia solani. Ann Appl Biol 161:247-254

Chen ZX, Feng ZM, Kang HX, Zhao JH, Chen TX, Li QQ, Gong HB, Zhang YF, Chen XB, Liu WD, Wang GL, Zuo SM (2019) Identification of new resistance loci against sheath blight disease in rice through genome-wide association study. Rice Sci 26:21-31

Chien CH, Chow CN, Wu NY, Chiang-Hsieh YF, Hou PF, Chang WC (2015) EXPath: a database of comparative expression analysis inferring metabolic pathways for plants. BMC Genomics 16:S6

Choi C, Hwang SH, Fang II R, Kwon Soon I, Park Sang R, Ahn I, Kim Jung B, Hwang DJ (2015) Molecular characterization of Oryza sativa WRKY6, which binds to W-box-like element 1 of the Oryza sativa pathogenesis-related (PR) 10a promoter and confers reduced susceptibility to pathogens. New Phytol 208:846-859

Cingolani P, Platts A, Wang LL, Coon M, Nguyen T, Wang L, Land SJ, Lu X, Ruden DM (2012) A program for annotating and predicting the effects of single nucleotide polymorphisms, SnpEff: SNPs in the genome of Drosophila melanogaster strain W1118; iso-2; iso-3. Fly (Austin) 6:80-92

Datta K, Tu J, Oliva N, Ona II, Velazhahan R, Mew TW, Muthukrishnan S, Datta SK (2001) Enhanced resistance to sheath blight by constitutive expression of infection-related rice chitinase in transgenic elite indica rice cultivars. Plant Sci 160:405-414

Datta K, Velazhahan R, Oliva N, Ona I, Mew T, Khush GS, Muthukrishnan S, Datta SK (1999) Over-expression of the cloned rice thaumatin-like protein (PR-5) gene in transgenic rice plants enhances environmental friendly resistance to Rhizoctonia solani causing sheath blight disease. Theor Appl Genet 98:1138-1145

Felsenstein J (1989) PHYLIP - phylogeny inference package (version 3.2). Cladistics 5:164-166

Gabriel SB, Schaffner SF, Nguyen H, Moore JM, Roy J, Blumenstiel B, Higgins J, DeFelice M, Lochner A, Faggart M, Liu-Cordero SN, Rotimi C, Adeyemo A, Cooper R, Ward R, Lander ES, Daly MJ, Altshuler D (2002) The structure of haplotype blocks in the human genome. Science 296:2225-2229

Han YP, Xing YZ, Chen ZX, Gu SL, Pan XB, Chen XL, Zhang QF (2002) Mapping QTLs for horizontal resistance to sheath blight in an elite rice restorer line, Minghui 63. Yi Chuan Xue Bao 29:622-626
Hossain MK, Jena KK, Bhuiyan MA, Wickneswari R (2016) Association between QTLS and morphological traits toward sheath blight resistance in rice (Oryza sativa L.). Breed Sci 66:613-626

IRRI (2002) Standard evaluation system for rice. International Rice Research Institute, Manila

Jia L, Yan W, Zhu C, Agrama HA, Jackson A, Yeater K, Li X, Huang B, Hu B, McClung A, Wu D (2012) Allelic analysis of sheath blight resistance with association mapping in rice. PLoS One 7:e32703

Kang H, Wang Y, Peng S, Zhang Y, Xiao Y, Wang D, Qu S, Li Z, Yan S, Wang Z, Liu W, Ning Y, Korniliev P, Leung H, Mezey J, McCouch SR, Wang GL (2016) Dissection of the genetic architecture of rice resistance to the blast fungus Magnaporthe oryzae. Mol Plant Pathol 17:959-972

Kang HM, Sul JH, Service SK, Zaitlen NA, Kong SY, Freimer NB, Sabatti C, Eskin E (2010) Variance component model to account for sample structure in genome-wide association studies. Nat Genet 42:348-354

Karmakar S, Molla KA, Chanda PK, Sarkar SN, Datta SK, Datta K (2016) Green tissue-specific co-expression of chitinase and oxalate oxidase 4 genes in rice for enhanced resistance against sheath blight. Planta 243:115-130

Karmakar S, Molla KA, Das K, Sarkar SN, Datta SK, Datta K (2017) Dual gene expression cassette is superior than single gene cassette for enhancing sheath blight tolerance in transgenic rice. Sci Rep 7:7900

Kawahara Y, de la Bastide M, Hamilton JP, Kanamori H, McCombie WR, Ouyang S, Schwartz DC, Tanaka T, Wu J, Zhou S, Childs KL, Davidson RM, Lin H, Quesada-Ocampo L, Vaillancourt B, Sakai H, Lee SS, Kim J, Numa H, Itoh T, Buell CR, Matsumoto T (2013) Improvement of the Oryza sativa Nipponbare reference genome using next generation sequence and optical map data. Rice (N Y) 6:4

Kouzai Y, Kimura M, Watanabe M, Kusunoki K, Osaka D, Suzuki T, Matsui H, Yamamoto M, Ichinose Y, Toyoda K, Matsuura T, Mori IC, Hirayama T, Minami E, Nishizawa Y, Inoue K, Onda Y, Mochida K, Noutoshi Y (2018) Salicylic aciddependent immunity contributes to resistance against Rhizoctonia solani, a necrotrophic fungal agent of sheath blight, in rice and Brachypodium distachyon. New Phytol 217:771-783

Kunihiro Y, Qian Q, Sato H, Teng S, Zeng DL, Fujimoto K, Zhu LH (2002) QTL analysis of sheath blight resistance in rice (Oryza sativa L.). Acta Genet $\operatorname{Sin} 29$ : 50-55

Kunst L, Samuels AL (2003) Biosynthesis and secretion of plant cuticular wax. Prog Lipid Res 42:51-80

Lee HI, Leon J, Raskin I (1995) Biosynthesis and metabolism of salicylic acid. Proc Natl Acad Sci U S A 92:4076-4079

Li F, Cheng L, Xu M, Zhou Z, Zhang F, Sun Y, Zhou Y, Zhu L, Xu J, Li Z (2009) QTL mining for sheath blight resistance using the backcross selected introgression lines for grain quality in rice. Acta Agron Sin 35:1729-1737

Li MX, Yeung JM, Cherny SS, Sham PC (2012) Evaluating the effective numbers of independent tests and significant p-value thresholds in commercial genotyping arrays and public imputation reference datasets. Hum Genet 131 $747-756$

Li Z, Pinson SR, Marchetti MA, Stansel JW, Park WD (1995) Characterization of quantitative trait loci (QTLs) in cultivated rice contributing to field resistance to sheath blight (Rhizoctonia solani). Theor Appl Genet 91:382-388

Lin W, Anuratha CS, Datta K, Potrykus I, Muthukrishnan S, Datta SK (1995) Genetic engineering of Rice for resistance to sheath blight. Bio-Technology 13:686

Liu G, Jia Y, Correa-Victoria FJ, Prado GA, Yeater KM, McClung A, Correll JC (2009) Mapping quantitative trait loci responsible for resistance to sheath blight in rice. Phytopathology 99:1078-1084

Liu X, Huang M, Fan B, Buckler ES, Zhang Z (2016) Iterative usage of fixed and random effect models for powerful and efficient genome-wide association studies. PLoS Genet 12:e1005767

Mao B, Liu X, Hu D, Li D (2014) Co-expression of RCH10 and AGLU1 confers rice resistance to fungal sheath blight Rhizoctonia solani and blast Magnorpathe oryzae and reveals impact on seed germination. World J Microbiol Biotechnol 30:1229-1238

Marshall DS, Rush MC (1980) Infection cushion formation on rice sheaths by Rhizoctonia solani. Phytopathology 70:947-950

Molla KA, Karmakar S, Chanda PK, Sarkar SN, Datta SK, Datta K (2016) Tissuespecific expression of Arabidopsis NPR1 gene in rice for sheath blight resistance without compromising phenotypic cost. Plant Sci 250:105-114

Mutuku JM, Nose A (2012) Changes in the contents of metabolites and enzyme activities in rice plants responding to Rhizoctonia solani Kuhn infection: activation of glycolysis and connection to phenylpropanoid pathway. Plant Cell Physiol 53:1017-1032 
Peng X, Hu Y, Tang X, Zhou P, Deng X, Wang H, Guo Z (2012) Constitutive expression of rice WRKY30 gene increases the endogenous jasmonic acid accumulation, PR gene expression and resistance to fungal pathogens in rice. Planta 236:1485-1498

Prasad B, Eizenga GC (2008) Rice sheath blight disease resistance identified in Oryza spp. accessions. Plant Dis 92:1503-1509

Purcell S, Neale B, Todd-Brown K, Thomas L, Ferreira MA, Bender D, Maller J, Sklar P, de Bakker PI, Daly MJ, Sham PC (2007) PLINK: a tool set for whole-genome association and population-based linkage analyses. Am J Hum Genet 81:559-575

Qin BX, Tang D, Huang J, Li M, Wu XR, Lu LL, Wang KJ, Yu HX, Chen JM, Gu MH, Cheng ZK (2011) Rice OsGL1-1 is involved in leaf cuticular wax and cuticle membrane. Mol Plant 4:985-995

Rakwal R, Yang G, Komatsu S (2004) Chitinase induced by jasmonic acid, methyl jasmonate, ethylene and protein phosphatase inhibitors in rice. Mol Biol Rep 31:113-119

Richa K, Tiwari IM, Devanna BN, Botella JR, Sharma V, Sharma TR (2017) Novel chitinase gene LOC_Os11g47510 from indica rice Tetep provides enhanced resistance against sheath blight pathogen Rhizoctonia solani in rice. Front Plant Sci 8:596

Savary S, Castilla NP, Elazegui FA, McLaren CG, Ynalvez MA, Teng PS (1995) Direct and indirect effects of nitrogen supply and disease source structure on rice sheath blight spread. Phytopathology 85:959-965

Seo PJ, Lee SB, Suh MC, Park MJ, Go YS, Park CM (2011) The MYB96 transcription factor regulates cuticular wax biosynthesis under drought conditions in Arabidopsis. Plant Cell 23:1138-1152

Shin JH, Blay S, McNeney B, Graham J (2006) LDheatmap: an R function for graphical display of pairwise linkage disequilibria between single nucleotide polymorphisms. J Stat Softw 16:9

Srinivasachary WL, Savary S (2011) Resistance to rice sheath blight (Rhizoctonia solani Kühn) [teleomorph: Thanatephorus cucumeris (a.B. frank) Donk.] disease: current status and perspectives. Euphytica 178:1-22

Taguchi-Shiobara F, Ozaki H, Sato H, Maeda H, Kojima Y, Ebitani T, Yano M (2013) Mapping and validation of QTLs for rice sheath blight resistance. Breed Sci 63:301-308

Tian T, Liu Y, Yan H, You Q, Yi X, Du Z, Xu W, Su Z (2017) agriGO v2.0: a GO analysis toolkit for the agricultural community, 2017 update. Nucleic Acids Res 45:W122-W129

Turner SD (2014) qqman: an R package for visualizing GWAS results using Q-Q and manhattan plots. bioRxiv. https://doi.org/10.1101/005165

Wang H, Meng J, Peng X, Tang X, Zhou P, Xiang J, Deng X (2015) Rice WRKY4 acts as a transcriptional activator mediating defense responses toward Rhizoctonia solani, the causing agent of rice sheath blight. Plant Mol Biol 89: 157-171

Wang W, Mauleon R, Hu Z, Chebotarov D, Tai S, Wu Z, Li M, Zheng T, Fuentes RR, Zhang F, Mansueto L, Copetti D, Sanciangco M, Palis KC, Xu J, Sun C, Fu B, Zhang H, Gao Y, Zhao X, Shen F, Cui X, Yu H, Li Z, Chen M, Detras J, Zhou Y, Zhang X, Zhao Y, Kudrna D, Wang C, Li R, Jia B, Lu J, He X, Dong Z, Xu J, Li Y, Wang M, Shi J, Li J, Zhang D, Lee S, Hu W, Poliakov A, Dubchak I, Ulat VJ, Borja FN, Mendoza JR, Ali J, Li J, Gao Q, Niu Y, Yue Z, Naredo MEB, Talag J, Wang X, Li J, Fang X, Yin Y, Glaszmann JC, Zhang J, Li J, Hamilton RS, Wing RA, Ruan J, Zhang G, Wei C, Alexandrov N, McNally KL, Li Z, Leung H (2018) Genomic variation in 3,010 diverse accessions of Asian cultivated rice. Nature 557:43-49

Wang X, Guan Y, Zhang D, Dong X, Tian L, Qu LQ (2017) A ß-Ketoacyl-CoA synthase is involved in rice leaf cuticular wax synthesis and requires a CER2LIKE protein as a cofactor. Plant Physiol 173:944-955

Wen ZH, Zeng YX, Ji ZJ, Yang CD (2015) Mapping quantitative trait loci for sheath blight disease resistance in Yangdao 4 rice. Genet Mol Res 14:1636-1649

Willocquet L, Noel M, Sackville Hamilton R, Savary S (2012) Susceptibility of rice to sheath blight: an assessment of the diversity of rice germplasm according to genetic groups and morphological traits. Euphytica 183:227-241

Wu B, Zhang B, Dai Y, Zhang L, Shang-Guan K, Peng Y, Zhou Y, Zhu Z (2012) Brittle culm15 encodes a membrane-associated chitinase-like protein required for cellulose biosynthesis in rice. Plant Physiol 159:1440-1452

Xie XW, Xu MR, Zang JP, Sun Y, Zhu LH, Xu JL, Zhou YL, Li ZK (2008) Genetic background and environmental effects on QTLs for sheath blight resistance revealed by reciprocal introgression lines in rice. Acta Agron Sin 34:1885-1893

Yadav S, Anuradha G, Kumar RR, Vemireddy LR, Sudhakar R, Donempudi K, Venkata D, Jabeen F, Narasimhan YK, Marathi B, Siddiq EA (2015) Identification of QTLs and possible candidate genes conferring sheath blight resistance in rice (Oryza sativa L.). Springerplus 4:175
Zeng Y, Ji Z, Ma L, Li X, Yang C (2011) Advances in mapping loci conferring resistance to rice sheath blight and mining Rhizoctonia solani resistant resources. Rice Sci 18:56-66

Zeng Y, Xia L, Wen Z, Ji Z, Zeng D, Qian Q, Yang C (2015) Mapping resistant QTLs for rice sheath blight disease with a doubled haploid population. J Integr Agric 14:801-810

Zhang F, Wu ZC, Wang MM, Zhang F, Dingkuhn M, Xu JL, Zhou YL, Li ZK (2017) Genome-wide association analysis identifies resistance loci for bacterial blight in a diverse collection of indica rice germplasm. PLoS One 12:e0174598

Zheng A, Lin R, Zhang D, Qin P, Xu L, Ai P, Ding L, Wang Y, Chen Y, Liu Y, Sun Z, Feng H, Liang X, Fu R, Tang C, Li Q, Zhang J, Xie Z, Deng Q, Li S, Wang S, Zhu J, Wang L, Liu H, Li P (2013) The evolution and pathogenic mechanisms of the rice sheath blight pathogen. Nat Commun 4:1424

Zou JH, Pan XB, Chen ZX, Xu JY, Lu JF, Zhai WX, Zhu LH (2000) Mapping quantitative trait loci controlling sheath blight resistance in two rice cultivars (Oryza sativa L.). Theor Appl Genet 101:569-573

Zuo S, Yin Y, Pan C, Chen Z, Zhang Y, Gu S, Zhu L, Pan X (2013) Fine mapping of qSB-11(LE), the QTL that confers partial resistance to rice sheath blight. Theor Appl Genet 126:1257-1272

Zuo S, Zhang L, Wang H, Yin Y, Zhang Y, Chen Z, Ma Y, Pan X (2008) Prospect of the QTL-qSB-9Tq utilized in molecular breeding program of japonica rice against sheath blight. J Genet Genomics 35:499-505

\section{Publisher's Note}

Springer Nature remains neutral with regard to jurisdictional claims in published maps and institutional affiliations.

\section{Submit your manuscript to a SpringerOpen ${ }^{\circ}$ journal and benefit from:}

- Convenient online submission

- Rigorous peer review

- Open access: articles freely available online

High visibility within the field

- Retaining the copyright to your article

Submit your next manuscript at $\boldsymbol{\nabla}$ springeropen.com 